\section{Effects of Carbon Tetrachloride on Mitosis}

We have observed that carbon tetrachloride produces striking effects on Allium cepa, Allium sativum and Zaphranthus sp. root tips. Root tips were treated for five minutes to a half-hour in carbon tetrachloride and later transferred to normal nutrient medium and fixed at various intervals from half an hour to six hours. Among the prominent changes taking place in the resting nucleus is a dissolution of the nuclear membrane and an outflow of its contents. Similar observations have been made in root tips of Allium cepa treated with chloroform ${ }^{1}$ and potassium cyanidea. It would appear that the lipoid layer of the nuclear membrane is either dissolved or rendered weak by the chemicals, in consequence of which the nuclear contents flow out. After treatment for $15 \mathrm{~min}$. with carbon tetrachloride, resting and premitotic nuclei break up. A number of chemicals are known to produce this effect, for example, urethane ${ }^{3}$ and nitrogen mustard“. Perhaps the most characteristic changes brought about by carbon tetrachloride are dissolution of chromatin in the resting nucleus and disorganization of the chromosome arms in the premitotic stages, resulting in their progressively negative reaction to the Feulgen reagent.

Mitotic stages show even more striking effects. One of the commonest is the occurrence of sticky bridges in anaphase where, due to decrease in viscosity of the matrix, the distal ends of chromosomes fail to separate ${ }^{5}$. Bridge formation has been reported as a result of the action of chloroform ${ }^{1}$, ethylene glycolo and nitrogen mustard ${ }^{7}$ among others. Metaphase chromosomes tend to become more or less despiralized, acquire side loops and resemble the 'lampbrush' chromosomes. Levan and Wangenheim ${ }^{2}$ have observed a similar phenomenon in onion root tips treated with potassium cyanide. Evidently the chemical affects spiralization and induces the material of the chromosome to flow out and form loops. Delayed separation of chromatids as well as chromo. some fragmentation are also common in material treated with carbon tetrachloride. The spindle, too, is affected, and results in irregular and unequal segregation of chromosomes and in polyploid nuelei. B. R. Seshachar Parvathi K. Nambiar

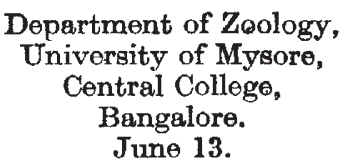

1 Steinigger, E., and Levan, A., Hereditas, 33, 552 (1947).

'Levan, A., and Wangenheim, K. H., Hereditas, 38, 297 (1952).

- Cornman, I., Inter. Rev. Cytol., 3, 113 (1954).

- Freidenwald, J. S., Ann. N.Y. Acad. Sci., S1, 1432 (1951).

- Varaama, A.s Hereditas, 33, 191 (1947).

- Ostergren, G., Bereditas, 80, 213 (1944).

'Darlington, C. D., and Koller, P. C., Heredity, 1, 187 (1947).

\section{Urinary Estrogens in the Buffalo as measured Chemically}

A KNOWLEDGe of the level and the rate at which hormones are excreted is of considerable academic value for diagnostic and therapentic purposes. The determination of cestrogen activity in the excreta is limited by the fact that these hormones are eliminated from the body in biologically active and inactive forms, thus necessitating chemical methods of measurement. The present work, consisting of two experiments, was carried out to ascertain the level and the rate at which urinary cestrogens are excreted in the buffalo and their chemical form.

Clean twenty-four hour specimens of urine were collected from four buffaloes during the fourth month of pregnancy, acidified with concentrated hydrochloric acid and boiled under reflux for $15 \mathrm{~min}$., then extracted four times with one-fourth volume peroxide-free ethyl ether. The extract was evaporated and the residue taken up in a minimal amount of ethanol. Each alcoholic extract was added to $300 \mathrm{ml}$. of toluene and then fractionated into neutral and phenolic fractions. The phenolic fraction was chromatographed and further fractionated by the use of Girard's reagent $T$ and phthalic anhydride ${ }^{1,2}$.

To determine the rate at which administered œstrogens are excreted in the urine, an ovariectomized buffalo was injected intramuscularly with $10 \mathrm{mgm}$. of cestradiol monobenzoate in microcrystal. line suspension, and $10 \mathrm{mgm}$. and $60 \mathrm{mgm}$. of cestradiol dipropionate dissolved in oil. Periods of seven days elapsed between the three different treatments, and urine samples were collected every six hours during the following $24 \mathrm{hr}$. Estimations of cestrogen were based on a physiochemical colori. metry ${ }^{2}$.

When the urinary cestrogens were fractionated, cestradiol and œstrone were found whereas oestriol was absent. The titres found for urinary costrogens (Table 1) were too low to have any quantitative significance. The pregnanediol titres, although incomplete, were of a very low order.

Table 1. Gestroghn and Pregnanediol Tutres excreted PeR

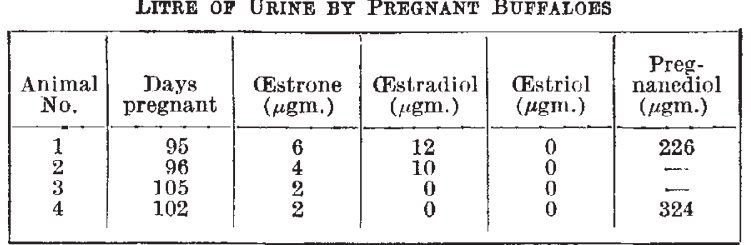

In the second experiment, only 0.5 per cent of the cestrogen injected in the third treatment was recovered in the urine specimen collected during the first six hours; while the first and second injections were not followed by any excretion of urinary cestrogens. CEstrous manifestations appeared after the dipropionate injections only.

Technical assistance given by Dr. Benjamin F. Stimmel, Rees-Staly Clinic Research Foundation, San Diego, California, is acknowledged. Thanks are due to the Ciba Foundation for providing hormone preparations. This work was carried out at the Agricultural Chemical Section, Giza, Egypt.

Faculty of Agriculture,

\section{E. S. E. HAFEZ*}

University of Cairo.

\section{T. ATTAR}

Agricultural Chemical Section, Orman, Giza.

- Present address : Worcester Foundation for Experimental Biology. Shrewsbury, Mass., U.S.A.

1 Stimmel, B. F., Randolf, M. A., John, D., and Conn, W. M., J. Clin. Endocrin., 12, 371 (1952). Stimmel, B. F., and May, J. A., ibid., II, 408 (1051).

- Cohen, H., and Bates, R. W., J. Clin. Endocrin., 7, 701 (1947); Endocrin., 44, 317 (1948). 